\title{
ATOMIC DATA AND SPECTRAL MODEL FOR Fe III
}

\author{
Manuel A. Bautista ${ }^{1}$, Connor P. Ballance ${ }^{2}$, and Pascal Quinet ${ }^{3,4}$ \\ ${ }^{1}$ Department of Physics, Western Michigan University, Kalamazoo, MI 49008, USA; manuel.bautista@wmich.edu \\ 2 Department of Physics, Auburn University, Auburn, AL 36849, USA \\ ${ }^{3}$ Astrophysique et Spectroscopie, Université de Mons-UMONS, B-7000 Mons, Belgium \\ ${ }^{4}$ IPNAS, B15 Sart Tilman, Université de Liège, Belgium \\ Received 2010 April 5; accepted 2010 July 1; published 2010 July 16
}

\begin{abstract}
We present new atomic data (radiative transitions rates and collision strengths) from large-scale calculations and a non-LTE spectral model for Fe III. This model is in very good agreement with observed astronomical emission spectra, in contrast with previous models that yield large discrepancies in observations. The present atomic computations employ a combination of atomic physics methods, e.g., relativistic Hartree-Fock, the Thomas-Fermi-Dirac potential, and Dirac-Fock computation of $A$-values and the $R$-matrix with intermediate coupling frame transformation and the Dirac $R$-matrix. We study advantages and shortcomings of each method. It is found that the Dirac $R$-matrix collision strengths yield excellent agreement with observations, much improved over previously available models. By contrast, the transformation of the LS-coupling $R$-matrix fails to yield accurate effective collision strengths at around $10^{4} \mathrm{~K}$, despite using very large configuration expansions, due to the limited treatment of spin-orbit effects in the near-threshold resonances of the collision strengths. The present work demonstrates that accurate atomic data for low-ionization iron-peak species are now within reach.
\end{abstract}

Key words: atomic data - circumstellar matter - H II regions - ISM: atoms - quasars: absorption lines - quasars: emission lines

\section{INTRODUCTION}

Reliable non-local thermodynamic equilibrium modeling of opacities and spectra of low-ionization stages of $\mathrm{Fe}$ and iron peak species is of paramount importance for 21st century astrophysics owing to various fundamental research areas that depend on these models. Such models require accurate transition rates (A-values) and effective collision strengths for electron impact excitation. These data sets have been difficult to obtain theoretically despite extensive efforts over many years (e.g., Bautista \& Pradhan 1998 and references therein), while experimental determinations remain very sparse or inexistent.

The Fe III spectrum is prominent in various galactic sources such as HiI regions, planetary nebulae, and Herbig-Haro objects (e.g., Mesa-Delgado et al. 2009), stars like $\eta$ Carinae (Johansson et al. 2000), and extragalactic objects such as active galactic nuclei (Laor et al. 1997; Vertergaard \& Wilkes 2001). These spectra are potentially useful in diagnosing the physical conditions of the plasmas and determining the gas phase Fe component. However, there are still shortcomings in our ability to interpret the observed spectra. Most quantitative analyses of Fe III spectra reported so far have relied on the electron impact excitation rates of Zhang (1996) and A-values for forbidden transitions of either Nahar \& Pradhan (1996) or Quinet (1996). While the collisional data accounted for all levels of the $3 d^{6}, 3 d^{5} 4 s$, and $3 d^{5} 4 p$ configurations, the available radiative data for dipole forbidden transitions comprise only the $3 d^{6}$ levels (see Bautista \& Pradhan 1998), leaving out important transitions among $3 d^{6}$ and $3 d^{5} 4 s$ levels. Moreover, these transitions are needed in modeling of fluorescent excitation mechanisms (Johansson et al. 2000).

The accuracy of current models for Fe III is questionable. When these are applied to analyze spectra of the Orion nebulae, the best studied nebula $\mathrm{H}$ II region, they yield great scatter among line ratio diagnostics and derive abundances from different lines. Furthermore, when these data are used in deriving the $\mathrm{Fe}$ abundance from [Fe III] lines, this differs by a factor of $\sim 4$ from abundances derived from [Fe IV] lines (Rodriguez \& Rubin 2005). Moreover, recent calculations of LS-coupling collision strengths by McLaughlin et al. $(2002,2007)$ yield significant differences with respect to those of Zhang (1996). Unfortunately, McLaughlin et al. provide no fine-structure data that can be used in spectral models.

We have carried out extensive calculations using several independent atomic structure codes. This multi-platform approach has proven successful in our previous studies of the $\mathrm{K}$ shells of $\mathrm{Fe}, \mathrm{O}, \mathrm{Ne}, \mathrm{Mg}, \mathrm{Si}, \mathrm{S}, \mathrm{Ar}, \mathrm{Ca}$, and $\mathrm{Ni}$ (e.g., Bautista et al. 2003; García et al. 2005; Palmeri et al. 2003b, 2003a). This allows for consistency checks and intercomparison. For calculations of atomic structure and $A$-values, we use the semi-relativistic Hartree-Fock (HFR) code of Cowan (1981), the multi configuration Dirac-Fock method in the code GRASP (Dyall et al. 1989; Parpia et al. 1996), and the central Thomas-Fermi-Dirac-Amaldi (TFDA) potential in AUTOSTRUCTURE (Badnell 1986, 1997). HFR provides selfconsistent optimization for the ground and lowest configurations and allows for semi-empirical corrections of the radial integral. The disadvantage, though, is that for complex systems like the present one the method converges only with a small number of configurations. GRASP is a fully relativistic code that can yield highly optimized wavefunctions, but for complex near-neutral systems it converges only for small numbers of configurations. AUTOSTRUCTURE is very efficient at treating large configuration expansions and configuration interaction $(\mathrm{CI})$ effects, but uses more approximate orbitals.

The $R$-matrix scattering calculations are carried out within two different coupling schemes. The first method (RM+ICFT hereafter) consists in the intermediate coupling frame transformation method (ICFT; Griffin et al. 1998) built upon the traditional LS $R$-matrix package (Berrington et al. 1995). It allows for the computationally intensive inner region calculation to be carried out in the LS coupling with the inclusion of mass-velocity and Darwin terms, and level-to-level collision strengths only being acquired from the transformation of term-resolved $K / S$ 
matrices in the outer region. Arguably, the capability of the RM+ICFT method to include large CI expansions in the description of the target, and also large numbers of correlation terms in the scattering wavefunction, has the potential to yield accurate results. However, while ICFT properly transforms the background cross sections and resonances with large principal quantum numbers, the positions of low $n$ resonances that lie within the $R$-matrix box are not corrected. Moreover, the large imbalance in the number of terms included in close coupling expansion and those used in the target description also has the potential to lead to spurious resonance structure.

Second, we employed a modified version of the DiracCoulomb $R$-matrix scattering package DARC (Ait-Tahar et al. 1996) and carried out the calculation in the JJ coupling in both the inner and outer regions. The availability of modern parallel supercomputers has removed many of the reservations of carrying out large-scale $R$-matrix calculations with DARC (e.g., Ballance et al. 2009).

The rest of this Letter is structured as follows. In Section 2, we describe the calculations of transition rates for dipole forbidden transitions. In Section 3, we present our calculations of collision strengths. In Section 4, we build a spectral model for the ion and compare its predictions for relative line intensities with observation of the well-known Orion nebula. Finally, discussion and conclusions are given in Section 5.

\section{RADIATIVE CALCULATIONS}

We compute radiative rates ( $A$-values) for dipole forbidden transition among the levels of $3 d^{6}$ and $3 d^{5} 4 s$ configurations using the codes AUTOSTRUCTURE and HFR.

AUTOSTRUCTURE is an atomic structure package written by Badnell $(1986,1997)$ initially based upon an earlier structure program SUPERSTRUCTURE (Eissner et al. 1974), which computes fine-structure level energies and radiative rates in a Breit-Pauli relativistic framework. Single-electron orbitals are constructed by diagonalizing the non-relativistic Hamiltonian, based on the TFDA model potential (Eissner \& Nussbaumer 1969). Scaling parameters for the potential are variationally optimized by minimizing LS term energies.

We performed calculations with various different configuration expansions, starting with models similar to those of previously published work and then evolving to larger expansions. Our final expansion includes 36 configurations, based on the 16configuration expansion of Zhang (1996), but adding pseudoorbitals $4 f, 5 s, 5 p$, and $5 d$ and various single and double promotions out of the $3 s$ and $3 p$ orbitals. A full description of the target will be given in a future publication. The orbitals were optimized minimizing the energies of the lowest 40 terms of the $3 d^{6}$ and $3 d^{5} 4 s$, and $3 d^{5} 4 p$ configurations. Fine-tuning of the wavefunctions was performed by means of term energy corrections. When comparing the predicted energy levels with experimental values (Ekberg 1993), the agreement is typically within $\sim 10 \%$ for the lowest terms up to about $0.55 \mathrm{Ry}$ and better for higher terms.

HFR uses the suite of codes of Cowan (1981) within the framework of the semi-relativistic HFR method. CI was retained among the following 12 configurations: $3 d^{6}, 3 d^{5} 4 s, 3 d^{5} 5 s$, $3 d^{5} 4 \mathrm{~d}, 3 d^{4} 4 s^{2}, 3 d^{4} 4 p^{2}, 3 d^{4} 4 d^{2}, 3 d^{4} 4 s 4 \mathrm{~d}, 3 d^{4} 4 s 5 s, 3 s 3 p^{6} 3 d^{7}$, $3 s 3 p^{6} 3 d 4 s$, and $3 s 3 p^{6} 3 d^{5} 4 s^{2}$. This configuration list considerably extends that of Quinet (1996). In addition to the explicit introduction of $\mathrm{CI}$, the interactions with more distant configurations were simulated through semi-empirical adjustment of the Slater parameters and the inclusion of additional effective parameters, such as $\alpha$ and $\beta$, associated with the excitations out of

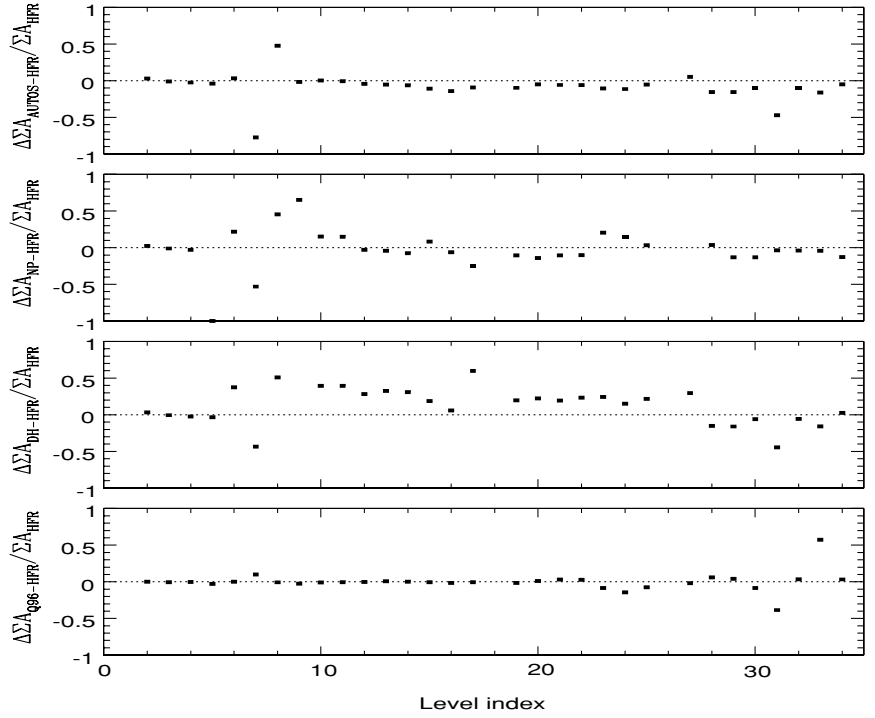

Figure 1. Comparison between present HFR results $\left(\sum A_{\mathrm{HFR}}\right)$ and those of Quinet (1996, $\sum A_{Q 96}$ ), SUPERSTRUCTURE calculations of Nahar \& Pradhan $\left(1996, \sum A_{\mathrm{NP}}\right)$ and Deb \& Hibbert (2009). In each panel, the $y$-axis gives the fractional differences with respect to the present HFR results, $\Delta \sum A_{\mathrm{X}-\mathrm{HFR}}=$ $\left(\sum A_{X}-\sum A_{\mathrm{HFR}}\right)$. The $x$-axis of the plots corresponds to the level indexes, which are assigned by strict increasing energy ordering. Levels 18 and 26 are missing from the figures because they belong to the $3 d^{5} 4 s$ configuration.

the $3 s$ and $3 p$ subshells into the $3 d$ (Trees 1951a, 1951b; Racah 1952). The fitting procedure was applied to the $3 d^{6}$ and $3 d^{5} 4 s$ configurations with the experimental energy levels published by Ekberg (1993). The ab initio HFR values for the Slater parameters within configurations other than those included in the fitting procedure and for the $\mathrm{CI}$ integrals, $R^{k}$, were scaled down by a factor of 0.90 as recommended by Cowan (1981), while the ab initio values of all the spin-orbit integrals, computed by the Blume-Watson method, were used without scaling.

In Figure 1 we compare the results of the present HFR and AUTOSTRUCTURE calculations. We also compare these values with those of Quinet (1996), Nahar \& Pradhan (1996), and Deb \& Hibbert (2009). The comparisons are made in terms of $\sum_{i>j} A_{i}$ which is of more practical interest than individual $A$-values. This is because $\sum_{i>j} A_{i}$ values are weighted toward the strongest transitions, which dominate the de-excitation of levels as well as the observed spectra. The comparison is for levels of the $3 d^{6}$ configuration, which are the only ones reported in previous publications. In comparing the present results with those of Nahar \& Pradhan (1996) one finds large differences for levels of the ${ }^{3} \mathrm{P}$ and ${ }^{3} \mathrm{H}$ terms and a dispersion of $20 \%-30 \%$ for the rest. There is significant scatter $(\sim 50 \%)$ between the results of all other calculations and those of Deb \& Hibbert (2009), who used the CIV 3 package. The only $A$-values in agreement are those among levels of the a ${ }^{5} D$ ground term (see Section 4). When comparing the present results with those of Quinet (1996), also from HFR, one finds excellent agreement for all but four levels. There is also a good agreement between the new AUTOSTRUCTURE and HFR ( 10\%-20\%), which suggests that the calculations have reached sufficient convergence for the most part. The only two problematic levels here are a ${ }^{3} H_{6}$ and a ${ }^{3} \mathrm{H}_{5}$ to which we have to assign a large uncertainty.

\section{COLLISION STRENGTHS}

Collision strengths for Fe III were computed by two different methods: RM+ICFT and DARC. For the RM+ICFT calculation 

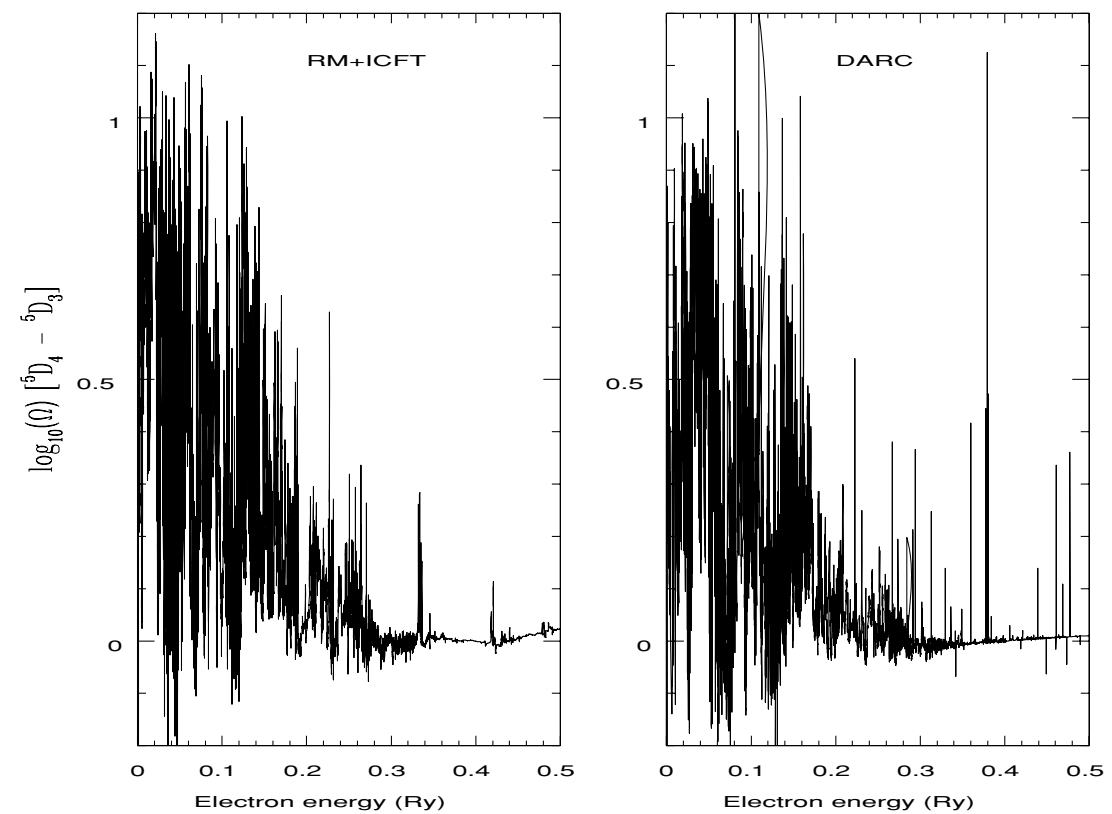

Figure 2. Collision strengths for the $3 d^{6}{ }^{5} D_{4}-{ }^{5} D_{3}$ transition computed with RM+ICFT and DARC.

we use the orbitals from our AUTOSTRUCTURE calculation, retaining CI from all 36 configurations. Yet, due to computational constrains the close coupling expansion only includes $136 \mathrm{LS}$ terms ( 283 levels) from the $3 d^{6}, 3 d^{5} 4 s$, and $3 d^{5} 4 p$ configurations. The calculation explicitly includes partial waves from states with $L \leqslant 12$ and multiplicity $1,3,5$, and 7 . The final collision strengths are produced with an energy resolution of $6 \times 10^{-5}$ Ry. The computations were performed with the suite of parallel Breit Pauli $R$-matrix programs (BPRM; Mitnik et al. 2001, 2003; Ballance \& Griffin 2004).

The DARC calculation was based on target orbitals determined by the Dirac-Hartree-Fock (DHF) atomic structure package GRASP (Dyall et al. 1989; Parpia et al. 1996) within an extended average level approximation. The CI expansion of the target included the eight configurations $3 s^{2} 3 p^{6} 3 d^{6}, 3 s^{2} 3 p^{4} 3 d^{8}$, $3 p^{6} 3 d^{8}, 3 s^{2} 3 p^{5} 3 d^{7}, 3 s^{2} 3 p^{6} 3 d^{5} 4 s, 3 s^{2} 3 p^{6} 3 d^{5} 4 p, 3 s^{2} 3 p^{4} 3 d^{7} 4 s$, and $3 s^{2} 3 p^{4} 3 d^{7} 4 p$ for a total of 2468 levels. The accuracy of the target states could have been further improved by including the configurations $3 s^{2} 3 p^{6} 3 d^{5} 4 d$ and $3 s^{2} 3 p^{4} 3 d^{7} 4 d$, but this was found to make the $R$-matrix calculation prohibitively large.

The scattering calculation was performed with the set of parallel DHF $R$-matrix programs (Ballance \& Griffin 2006; Badnell et al. 2004), which uses modified subroutines based upon the serial version of the DARC $\operatorname{code}^{5}$ and portions of the $R$-matrix programs. The close coupling expansion of the target included 322 levels arising from the configurations $3 s^{2} 3 p^{6} 3 d^{6}, 3 s^{2} 3 p^{6} 3 d^{5} 4 s$, and $3 s^{2} 3 p^{6} 3 d^{5} 4 p$. Fortunately, the last two configurations of our CI expansion do not support energy levels below 7 Ryd. All JП partial waves from $J=0$ to $J=29.5$ were included in the calculation, and contributions from the higher partial waves were estimated from a Burgess \& Tully (1978) top-up procedure. For partial waves up to 9.5 , we employed 11 basis orbitals for each continuum-electron angular momentum. This continuum basis was sufficient to span electron energies up to 3.0 Ryd.

We find that while RM+ICFT and DARC yield very similar background collision strengths, there are slight differences in the positions of the resonances near threshold. This is illustrated in

\footnotetext{
5 http://www.am.qub.ac.uk/DARC
}

Table 1

Maxwellian-averaged Collision Strengths at 10,000 K from the Ground Level $3 d^{6} D_{4}$ to the Excited Levels of $3 d^{6}$

\begin{tabular}{lccc}
\hline \hline Upper Level & RM+ICFT & DARC & Zhang \\
\hline${ }^{5} D_{3}$ & $4.57 \mathrm{E}+0$ & $2.54 \mathrm{E}+0$ & $2.92 \mathrm{E}+0$ \\
${ }^{5} D_{2}$ & $1.94 \mathrm{E}+0$ & $1.11 \mathrm{E}+0$ & $1.24 \mathrm{E}+0$ \\
${ }^{5} D_{1}$ & $8.79 \mathrm{E}-0$ & $5.33 \mathrm{E}-1$ & $5.95 \mathrm{E}-1$ \\
${ }^{5} D_{0}$ & $2.51 \mathrm{E}-1$ & $1.60 \mathrm{E}-1$ & $1.80 \mathrm{E}-1$ \\
${ }^{3} 2_{2}$ & $7.14 \mathrm{E}-1$ & $7.14 \mathrm{E}-1$ & $5.80 \mathrm{E}-1$ \\
${ }^{3} \mathrm{P}_{1}$ & $1.84 \mathrm{E}-1$ & $1.96 \mathrm{E}-1$ & $1.65 \mathrm{E}-1$ \\
${ }^{3} 2_{0}$ & $3.83 \mathrm{E}-2$ & $3.25 \mathrm{E}-2$ & $2.13 \mathrm{E}-2$ \\
${ }^{3} \mathrm{H}_{6}$ & $2.66 \mathrm{E}+0$ & $1.21 \mathrm{E}+0$ & $1.34 \mathrm{E}+0$ \\
${ }^{3} H_{5}$ & $1.10 \mathrm{E}+0$ & $9.84 \mathrm{E}-1$ & $4.89 \mathrm{E}-1$ \\
${ }^{3} \mathrm{H}_{4}$ & $2.41 \mathrm{E}-1$ & $5.33 \mathrm{E}-1$ & $9.26 \mathrm{E}-2$ \\
${ }^{3} F 2_{4}$ & $1.47 \mathrm{E}+0$ & $4.54 \mathrm{E}-1$ & $1.07 \mathrm{E}+0$ \\
${ }^{3} \mathrm{~F}_{3}$ & $6.42 \mathrm{E}-1$ & $1.91 \mathrm{E}-1$ & $4.35 \mathrm{E}-1$ \\
${ }^{3} \mathrm{~F}_{2}$ & $2.11 \mathrm{E}-1$ & $1.73 \mathrm{E}-1$ & $1.57 \mathrm{E}-1$ \\
${ }^{3} G_{5}$ & $1.11 \mathrm{E}+0$ & $1.36 \mathrm{E}+0$ & $1.10 \mathrm{E}+0$ \\
${ }^{3} G_{4}$ & $1.24 \mathrm{E}+0$ & $1.11 \mathrm{E}+0$ & $4.28 \mathrm{E}-1$ \\
${ }^{3} G_{3}$ & $4.52 \mathrm{E}-1$ & $4.21 \mathrm{E}-1$ & $1.09 \mathrm{E}-1$ \\
\hline
\end{tabular}

Figure 2, where one can see that a dense resonance structure is shifted toward the threshold in the RM+ICFT calculation with respect to the DARC result. These resonances dominate the Maxwellian-averaged collision strengths at temperatures near $10^{4} \mathrm{~K}$, typical of photoionized plasmas. For this reason, the RM+ICFT Maxwellian-averaged values are systematically higher than those from DARC by as much as $\sim 50 \%$, as shown in Table 1 for a sample of transitions. Also, there are large differences between the present results and those of Zhang (1996) for many transitions. The calculations of Zhang were carried out in LS-coupling plus algebraic splitting of collision strengths to fine structure.

\section{COMPARISON WITH OBSERVED ASTRONOMICAL SPECTRA}

We benchmarked the quality of the various data sets against spectra of the Orion nebula by Mesa-Delgado et al. (2009). This deep, high-resolution spectrum shows 33 optical and 

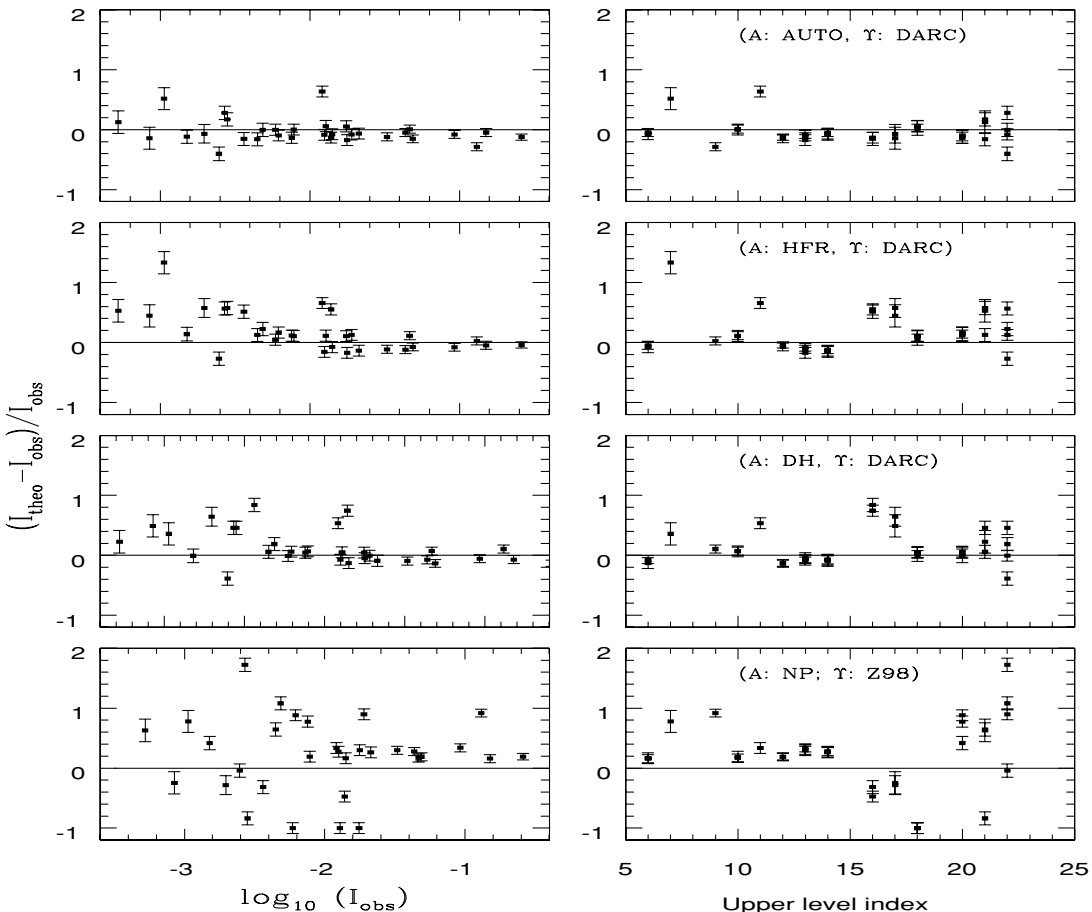

Figure 3. Comparison between observed and predicted line intensities for all lines measured in the "shock component" of the Orion Nebula. The left panels compare the lines intensities vs. the logarithm on the observed intensity, and in the right panels the comparison is shown against the levels index of the upper level of the line. From top to bottom, the first three rows are for theoretical intensities using the present DARC collision strengths and A-values from AUTOSTRUCTURE, HFR, and Deb and Hibbert (HD), respectively, while the lowest panels are based on theoretical intensities using collisional data from Zhang (1996) and $A$-values from Nahar \& Pradhan (1996). The upper panel shows the results from our present data.

near-infrared lines in a shocked gas region, "shock component," and 16 lines in a more diluted region, "nebular component." The electron densities, $n_{e}$, are diagnosed from lines of [O II], [S II], [Cl III ], and [Ar IV] in both regions as $2890 \pm 550 \mathrm{~cm}^{-3}$ and $17430 \pm 2500 \mathrm{~cm}^{-3}$, respectively. The electron temperature, $T_{e}$, in both regions is approximately $9000 \mathrm{~K}$.

We solve excitation equilibrium models for Fe III using the various sets of atomic data available adopting the values of $n_{e}$ and $T_{e}$ given above. The fluxes of theoretical lines are normalized to the total flux predicted over all observed lines. The fluxes of observed lines are also normalized to the sum of fluxes of all lines. Figure 3 shows the comparison between the theoretical and observed normalized intensities for the "shock component," which has the richest spectrum. The comparison is in terms of the fractional difference between theoretical intensities and observations. The error bars in this figure are the statistical errors of the observations.

We find that the previous model, which uses collision strengths from Zhang (1996) and $A$-values from Nahar \& Pradhan (1996), yields large scatter for all lines. The new collisional data from DARC in combination with any of the newer sets of $A$-values yield much better agreement with observations. When comparing line intensities versus the observed line intensity, one sees that the scatter increases toward the weakest lines, as expected from increasing theoretical and observational uncertainties for weaker lines. The comparisons of line intensities versus the upper level index of the line allow one to see the effects of different sets on the population of each excited level. When two or more lines are observed from the same level, the dispersion of these along the vertical direction indicates the uncertainties in the radiative branching ratios for the decay of the level. One can see that for levels 20,21 , and $22\left({ }^{3} D_{6}, 5,4\right)$ the Nahar \& Pradhan $A$-values yield considerable dispersion in branching ratios, unlike any of the other $A$-values data sets. For levels 16 and $17\left({ }^{3} G_{4,3}\right)$ the combination of DARC collision strengths and $A$-values from Deb \& Hibbert and the present HFR calculation yield line intensities overestimated by $\sim 60 \%$, while the present AUTOSTRUCTURE $A$-values yield good agreement with observations. For level $11\left({ }^{3} P_{0}\right)$ all the newer $A$-values yield overestimated uncertainties by $\sim 50 \%$, suggesting that the error could come from the collision strengths, unless it is in the measured line intensity. The predicted population of level $7\left({ }^{3} \mathrm{H}_{6}\right)$ seems too high in all models, but it is particularly problematic when using $A$-values from the present HFR calculation. The total radiative decay rate from this level as computed with HFR disagrees with the results of all other calculations. Thus, the observations seem to provide evidence against the HFR rates for this level.

When using the RM+ICFT collision strengths in the calculation of line intensities, these are considerably worse than those from the data of Zhang (1996). The large difference between the ICFT and DARC calculations is unexpected. There was an expectation that given the extensive CI description of the target the ICFT method would compare more favorably with observations than the DARC calculation, which uses only seven configurations. However, it appears that this relatively small DARC calculation gives sufficiently accurate collision strengths. While the background collision strengths are very similar among the ICFT and DARC calculations, this is not the case for nearthreshold resonances, which dominate the Maxwellian-averaged values for forbidden transitions. Representation of these resonances with RM+ICFT is fundamentally flawed because the transformation does not correct their positions by the spin-orbit coupling. Neither does the algebraic transformation used by Zhang. 


\section{DISCUSSION AND CONCLUSIONS}

We have carried out the most extensive and systematic calculation of radiative and collisional data for Fe III done up to the present. We employed a multi-method approach that allowed us to study the effects of different approximations and the advantages and shortcomings of each method.

In the calculation of $A$-values, we find convergence of the results in terms of methods and configuration expansions, the present ones being significantly larger than that in previous work. There is a general good agreement between the present AUTOSTRUCTURE and HFR results, despite significant discrepancies for a handful of levels. The present results agree within $\sim 20 \%$ with the results of Deb \& Hibbert (2009), but greater discrepancies are found with respect to those of Nahar \& Pradhan (1996). It is difficult to pick the most accurate of these calculations on the basis of the methods used alone (although see Deb \& Hibbert 2009). Though, comparisons between predicted line intensities and measured lines in the spectra of the Orion nebula seem to favor the present AUTOSTRUCTURE results over other calculations, and the $A$-values of Deb \& Hibbert compare better with observations than those from HFR.

Benchmarking of collision strengths computed in the LS coupling with transformation to an intermediate coupling and those in the $\mathrm{jJ}$ coupling sheds unexpected and important conclusions. Only calculations in $\mathrm{jJ}$ can accurately treat the near-threshold resonances that dominate thermally averaged collision strengths at typical temperatures of photoionized nebulae. In the case of $\mathrm{Fe}$ III, the most important effect on resonance positions comes from the spin-orbit coupling, and not so much from relativistic effects. In fact, algebraic splitting of LS collision strengths is practically as accurate as ICFT in terms of the background cross sections, but neither one can treat the near-threshold resonances. Moreover, we see that the results of the present very large RM+ICFT calculations are somewhat worse than those of Zhang (1996). This is because the higher orbitals in the present CI expansion lead to a larger $R$-matrix box and this yields even poorer treatment of near-threshold resonances and over a more extended energy region.

At this point, one may ask whether BPRM calculations, i.e., in the $\mathrm{jK}$ coupling, of collision strengths could be an alternative to the use of DARC. The answer is probably yes, although most BPRM packages available do not include two-body relativistic terms, but these should be unimportant here. However, the main difference between the two approaches will be in the quality of the atomic orbitals used. DARC uses orbitals from GRASP, which is fully relativistic, thus optimizes on the energy levels and yields two components to the radial functions of each orbital. This is ideal for representing strongly mixed levels. By contrast, AUTOSTRUCTURE orbitals to use in BPRM calculations are optimized on LS terms only, thus greater CI expansions are often needed to reach the same degree of accuracy given by GRASP.

The high accuracy of the present Fe III spectral model, which uses collisional data from DARC and $A$-values from AUTOSTRURE, is demonstrated by comparison with observed optical and near-infrared spectra of the Orion nebulae. In the absence of experimental determinations of atomic parameters, comparison with observed spectra is the best way to test the accuracy of the atomic models. The present comparisons demonstrate that the new atomic data are considerably better than previous results. Moreover, this study opens the door to the computation of long-awaited accurate models for lowly ionized iron-peak species.

We acknowledge financial support from grants from the NASA Astronomy and Physics Research and Analysis Program (award NNX09AB99G), the Space Telescope Science Institute (project GO-11745), US Department of Energy, and the Belgian FRS-FNRSs, and from the Belgian FRS-FNRS at which P.Q. is a Senior Research Associate. Most of the computations were carried out at the National Energy Research Scientific Computing Center in Oakland, California and at the National Center for Computational Sciences at Oak Ridge under the Teragrid program.

\section{REFERENCES}

Ait-Tahar, S., Grant, I. P., \& Norrington, P. H. 1996, Phys. Rev. A, 54, 3894 Badnell, N. R. 1986, J. Phys. B, 19, 3827

Badnell, N. R. 1997, J. Phys. B, 30, 1

Badnell, N. R., Berrington, K. A., Summers, H. P., O’Mullane, M. G., Whiteford, A. D., \& Ballance, C. P. 2004, J. Phys. B: At. Mol. Opt. Phys., 37, 4589

Ballance, C. P., \& Griffin, D. C. 2004, J. Phys. B: At. Mol. Opt. Phys., 37, 2943 Ballance, C. P., \& Griffin, D. C. 2006, J. Phys. B: At. Mol. Opt. Phys., 40, 3617 Ballance, C. P., Ludlow, J. A., Pindzola, M. S., \& Loch, S. D. 2009, J. Phys. B: At. Mol. Opt. Phys., 42, 5202

Bautista, M. A. 2004, A\&A, 420, 763

Bautista, M. A., Mendoza, C., Kallman, T. R., \& Palmeri, P. 2003, A\&A, 403, 339

Bautista, M. A., \& Pradhan, A. K. 1998, ApJ, 492, 650

Berrington, K. A., Eissner, W. B., \& Norrington, P. H. 1995, Comput. Phys. Commun., 92, 290

Blandford, R. D., \& Begelman, M. C. 2004, MNRAS, 349, 68

Burgess, A., \& Tully, J. A. 1978, J. Phys. B: At. Mol. Phys., 11, 4271

Cowan, R. D. 1981, The Theory of Atomic Structure and Spectra (Berkeley: Univ. California Press)

Deb, N. C., \& Hibbert, A. 2009, J. Phys. B: At. Mol. Opt. Phys., 42, 065003

Dyall, K. G., Grant, I. P., Jonhson, C. T., Parpia, F. A., \& Plummer, E. P. 1989, Comput. Phys. Commun., 55, 424

Eissner, W., Jones, M., \& Nussbaumer, H. 1974, Comput. Phys. Commun., 8, 270

Eissner, W., \& Nussbaumer, H. 1969, J. Phys. B, 2, 1028

Ekberg, J. O. 1993, A\&AS, 101, 1

García, J., Mendoza, C., Bautista, M. A., Gorczyca, T. W., Kallman, T. R., \& Palmeri, P. 2005, ApJS, 158, 68

Griffin, D. C., Badnell, N. R., \& Pindzola, M. S. 1998, J. Phys. B: At. Mol. Opt Phys., 31, 3713

Johansson, S., Zethson, T., Hartman, H., Ekberg, J. O., Ishibashi, K., Davidson, K., \& Gull, T. 2000, A\&A, 361, 977

Laor, A., Jannuzi, B. T., Green, R. F., \& Boroson, T. A. 1997, ApJ, 489, 656

McLaughlin, B. M., Scott, M. P., Sunderland, A. G., Noble, C. J., Burke, V. M., \& Burke, P. G. 2002, J. Phys. B, 35, 2755

McLaughlin, B. M., et al. 2007, At. Data Nucl. Data Tables, 93, 55

Mesa-Delgado, A., Esteban, C., García-Rojas, J., Luridiana Bautista, M., Rodríguez, M., López-Martín, L., \& Peimbert, M. 2009, MNRAS, 395, 855

Mitnik, D. M., Griffin, D. C., \& Badnell, N. R. 2001, J. Phys. B: At. Mol. Opt. Phys., 34, 4455

Mitnik, D. M., Griffin, D. C., Balance, C. P., \& Badnell, N. R. 2003, J. Phys. B: At. Mol. Opt. Phys., 36, 717

Nahar, S. N., \& Pradhan, A. K. 1996, A\&AS, 119, 509

Palmeri, P., Mendoza, C., Kallman, T. R., \& Bautista, M. A. 2003a, A\&A, 403, 1175

Palmeri, P., Mendoza, C., Kallman, T. R., Bautista, M. A., \& Mlendez, M. 2003b, A\&A, 410, 359

Parpia, F. A., Froese Fischer, C., \& Grant, I. P. 1996, Comput. Phys. Commun., 94, 249

Quinet, P. 1996, A\&AS, 116, 573

Racah, G. 1952, Phys. Rev., 85, 381

Rodríguez, M., \& Rubin, R. H. 2005, ApJ, 626, 900

Trees, R. E. 1951a, Phys. Rev., 83, 756

Trees, R. E. 1951b, Phys. Rev., 84, 1089

Vertergaard, M., \& Wilkes, B. J. 2001, ApJS, 134, 1

Zhang, H. L. 1996, A\&AS, 119, 523 